\title{
Endostatin improves cancer-associated systemic syndrome in a lung cancer model
}

\author{
XIA WANG ${ }^{*}$, RUI-YU ZHAN ${ }^{*}$, YU-YI WANG $^{*}$, XI YAN, DAN CAO, YAN LI, YI-QIN WANG and FENG LUO \\ Department of Medical Oncology, Cancer Center and State Key Laboratory of Biotherapy, \\ West China Hospital of Sichuan University, Chengdu, Sichuan 610041, P.R. China
}

Received July 7, 2014; Accepted February 18, 2015

DOI: $10.3892 / \mathrm{ol} .2015 .3049$

\begin{abstract}
Cancer-associated systemic syndrome (CASS) is characterized by a constellation of symptoms, including progressive weight loss, anemia, endocrine disorders, gastrointestinal dysfunction, muscle and adipose atrophy, hepatic peliosis and kidney failure. The present study assesses the effects of endostatin on CASS and any possible underlying mechanism in tumor-bearing mice. The results showed that the inoculation of Lewis lung carcinoma cells into mice led to CASS that was characterized by a notable decrease in body weight, severe anemia phenotype, disordered biochemistry, hepatosplenomegaly, and a marked increase in serum vascular endothelial growth factor (VEGF), tumor necrosis factor $\alpha$ and interleukin-6 (IL-6). The continuous injection of $10 \mathrm{mg} / \mathrm{kg} /$ day endostatin suppressed tumor growth and alleviated CASS in the tumor-bearing mice, as shown by weight gain, improvement in biochemistry and anemia, and the preservation of organ function. The effects of endostatin on CASS in the tumor-bearing mice were accompanied by the downregulation of serum VEGF and IL-6. Collectively, these findings indicate that endostatin improves CASS in tumor-bearing mice by decreasing the serum levels of VEGF and IL-6.
\end{abstract}

Correspondence to: Professor Feng Luo, Department of Medical Oncology, Cancer Center and State Key Laboratory of Biotherapy, West China Hospital of Sichuan University, 37 Guoxue Xiang Street, Chengdu, Sichuan 610041, P.R. China

E-mail: luofeng@medmail.com.cn

*Contributed equally

Abbreviations: CASS, cancer-associated systemic syndrome; LLC, Lewis lung carcinoma; VEGF, vascular endothelial growth factor; TNF- $\alpha$, tumor necrosis factor $\alpha$; IL, interleukin; HGB, hemoglobin; HCT, hematocrit; AST, aspartate aminotransferase; ALB, albumin; TP, total protein; $\mathrm{CHO}$, cholesterol; HDL, high-density lipoprotein; LDL, low-density lipoprotein; H\&E, hematoxylin and eosin

Key words: endostatin, Lewis lung carcinoma, cancer-associated systemic syndrome, angiogenic cytokines

\section{Introduction}

The majority of patients with an advanced malignancy develop a range of systemic disorders. Cancer-associated systemic syndrome (CASS) refers to the cluster of symptoms that typically includes fever, anemia, endocrine and neurological disorders, gastrointestinal dysfunction, adipose and muscle atrophy, hepatic peliosis, ascites and kidney failure $(1,2)$. The syndrome can also manifest as cachexia and paraneoplastic syndrome in severe cases $(1,3)$. CASS is the most common cause of cancer-related mortality, as it confers susceptibility to infections and other secondary pathologies on cancer patients and tumor-transplanted mice (4), in addition to reducing patient responsiveness to chemotherapy (5).

Angiogenic cytokines, including tumor necrosis factor $\alpha$ (TNF- $\alpha$ ), interleukin (IL)-1 and IL-6, contribute to CASS and poor survival $(6,7)$. Vascular endothelial growth factor (VEGF) is an angiogenic factor that has been characterized extensively and is expressed at high levels in the majority of tumors. In the local tumor environment, the protein modulates blood vessel growth, vascular permeability and vascular remodeling, while VEGF produced by tumors also accumulates in the circulation and induces CASS $(2,8)$.

Angiogenesis is an important process required for the growth and metastasis of malignant tumors. Antiangiogenic drugs, including sunitinib, bevacizumab and sorafenib, cause the modulation of tumor growth via vascular remodeling and regression, which improves progression-free survival (9). These drugs may modulate off-target vasculature in a range of organs and tissues. As a consequence of these off-tumor target effects, improvements in CASS and alterations in chemotoxic tolerance may increase overall survival in patients with cancer (9).

Endostatin, an endogenous angiogenesis inhibitor, inhibits VEGF-stimulated proliferation, migration and tube formation by suppressing the VEGF-induced tyrosine phosphorylation of KDR/Flk-1 [also known as VEGF receptor 2 (VEGFR-2)], overall VEGFR-2 expression, and the activation of extracellular signal-regulated kinases, p38 mitogen-activated protein kinase and AKT (10-12). Furthermore, endostatin suppresses the expression of VEGF, fibroblast growth factor, TNF- $\alpha$, matrix metalloproteinases and vascular cell adhesion molecule-1 (13). Therefore, we hypothesized that endostatin, as a broad-spectrum antiangiogenic agent, would exhibit anti-CASS effects. 
In the present study, an experiment was conducted in Lewis lung carcinoma (LLC) tumor-bearing mice to test this hypothesis and investigate any possible mechanisms.

\section{Materials and methods}

Cell lines and reagents. LLC cell lines were obtained from the American Type Culture Collection (Manassas, VA, USA), cultured in Dulbecco's modified Eagle's medium (Gibco BRL, Life Technologies, Grand Island, NY, USA) supplemented with $10 \%$ fetal bovine serum, and kept in a humidified $5 \%$ $\mathrm{CO}_{2}$ atmosphere at $37^{\circ} \mathrm{C}$. Recombinant human endostatin was obtained from Shandong Simcere Medgenn Bio-Pharmaceutical Co., Ltd. (Yantai, Shandong, China). A monoclonal goat anti-mouse cluster of differentiation (CD) 31 antibody (cat. no. sc-1506) was purchased from Santa Cruz Biotechnology Inc. (Dallas, TX, USA). The TNF- $\alpha$, VEGF and IL-6 concentrations were detected using an enzyme-linked immunosorbent assay (ELISA) kit (Neobioscience, Shenzhen, Guangdong, China). Tumor tissues from the animals were homogenized and solubilized in RIPA lysis buffer (Beyotime Institute of Biotechnology, Shanghai, China).

Tumor models and treatment protocol. Specific pathogen-free, male, C57BL/6 mice (5-6 weeks old) were purchased from the Laboratory Animal Center of Sichuan University (Chengdu, Sichuan, China) and maintained in pathogen-free conditions. All experiments were carried out in accordance with the guidelines approved by the Animal Care and Use Committee of Sichuan University. The experimental procedures and the animal use and care protocols were approved by the Committee on Ethical Use of Animals of West China Hospital of Sichuan University (Chengdu, China).

C57BL/6 mice were randomly distributed into 3 groups ( $\mathrm{n}=10 /$ group); the control group (NS group) was subcutaneously inoculated with $1 \times 10^{6}$ LLC cells, the endostatin group (ES group) was subcutaneously inoculated with $1 \times 10^{6}$ LLC cells, and the normal group consisted of non-tumor-bearing mice. The NS and normal groups received normal saline (NS), but the ES group received $10 \mathrm{mg} / \mathrm{kg}$ endostatin at 8 days post-implantation. After day $8,10 \mathrm{mg} / \mathrm{kg}$ endostatin or normal saline was intravenously administered daily (by caudal vein injection) for 12 days. Tumor volume was estimated every 3 days using the following formula: Tumor volume $=a \mathrm{x} \mathrm{b}^{2} / 2$, where $\mathrm{a}$ and $\mathrm{b}$ are the long and short tumor diameters, respectively. At 20 days post-implantation, the animals were sacrificed by the cervical dislocation method in order to harvest tissues and blood samples for further analysis.

Changes in body and organ weights. Body weight was measured every 3 days after tumor implantation. Tumors were removed in order to calculate body weight as the whole body weight minus the tumor weight. Organs were dissected and weighed for comparison.

Blood sample analysis. Blood samples were collected from the orbital sinus. Whole blood and serum samples were prepared in the presence or absence of the ethylenediaminetetraacetic acid anticoagulant. Hematological parameters, including hemoglobin (HGB) level, hematocrit (HCT) and red blood cell count, were determined in whole blood using the MEK6318K automatic blood analyzer (Nihon Kohden, Tokyo, Japan). Biochemical indicators of liver function and metabolism, including serum alanine aminotransferase, aspartate aminotransferase (AST), albumin (ALB), total protein (TP), glucose, triglycerides, cholesterol (CHO), high-density lipoprotein (HDL) and low-density lipoprotein (LDL), were determined using a Hitachi 7020 automatic biochemical analyzer (Hitachi High-Tech, Tokyo, Japan) according to the manufacturer's instructions.

Histological and immunohistochemical analysis. Tumor-bearing and control mice were sacrificed at day 20 post-implantation. Necropsies were performed, and various tissues and organs were removed, weighed and immediately fixed overnight in $4 \%$ paraformaldehyde. Samples of the tissues and organs were embedded in paraffin. Malignant and non-malignant paraffin-embedded tissues were sectioned to a $5-\mu \mathrm{m}$ thickness and stained with hematoxylin and eosin (H\&E). The microvessels were determined by CD31 immunostaining. Paraffin-embedded tissues sections were deparaffinized, rehydrated, incubated in $3 \% \mathrm{H}_{2} \mathrm{O}_{2}$ and blocked with $5 \%$ bovine serum albumin. The sections were then incubated with CD31 antibody (dilution, 1:400) at $4^{\circ} \mathrm{C}$ overnight, followed by incubation with biotinylated polyclonal rabbit anti-goat antibody (dilution, 1:200; cat. no. ab124055; Beyotime Institute of Biotechnology). Peroxidase activity was visualized using a 3,3'-diaminobenzidine substrate kit (CellChip Biotechnology, Beijing, China). Sections were counterstained with hematoxylin. The slides were examined using an Eclipse E600 microscope (Nikon, Tokyo, Japan).

ELISA. Blood samples were collected from all mice after treatment using non-anticoagulation infertile tubes. Samples were centrifuged at $2,800 \mathrm{xg}$ for $10 \mathrm{~min}$ at $4^{\circ} \mathrm{C}$, and separated serum samples were stored at $-80^{\circ} \mathrm{C}$ until use. The tumor tissues from the animals were homogenized and solubilized in lysis buffer using a commercial kit. Samples were centrifuged at $11,000 \mathrm{xg}$ for $30 \mathrm{~min}$ at $4^{\circ} \mathrm{C}$, and the separated supernatants were stored at $-80^{\circ} \mathrm{C}$ until use. TNF- $\alpha$, VEGF and IL-6 (serum and tumor samples) were assessed using commercial mouse ELISA kits according to the manufacturer's instructions. The colorimetric reaction was monitored at $450 \mathrm{~nm}$ using a Benchmark microplate reader (Benchmark Electronics, Angleton, TX, USA).

Statistical analysis. Data are expressed as the mean \pm standard deviation. Statistical analyses were performed using SPSS software (version 16.0; SPSS, Inc., Chicago, IL, USA). Between-group statistical significance was determined using a one-way analysis of variance, and a least significant difference test was applied for multiple comparisons. $\mathrm{P}<0.05$ was used to indicate a statistically significant difference.

\section{Results}

Anticancer effects of endostatin. The in vivo results indicated that the tumors initially became palpable around 7 days after inoculation. The long and short tumor diameters were estimated after 8 days (Fig. 1A). From day 14 onwards, the tumor volume of the ES group was significantly lower than 
A

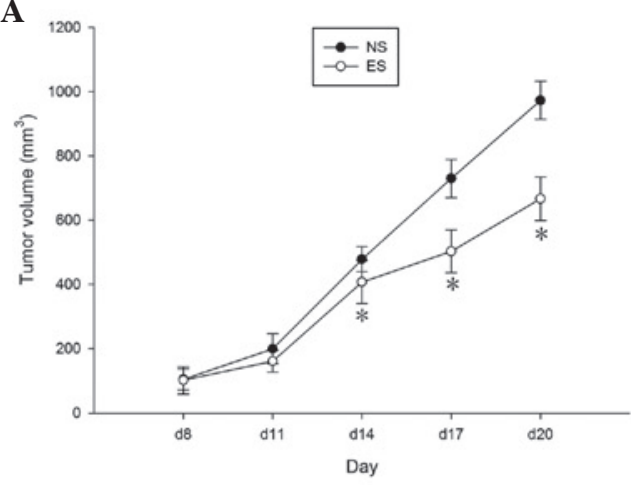

C

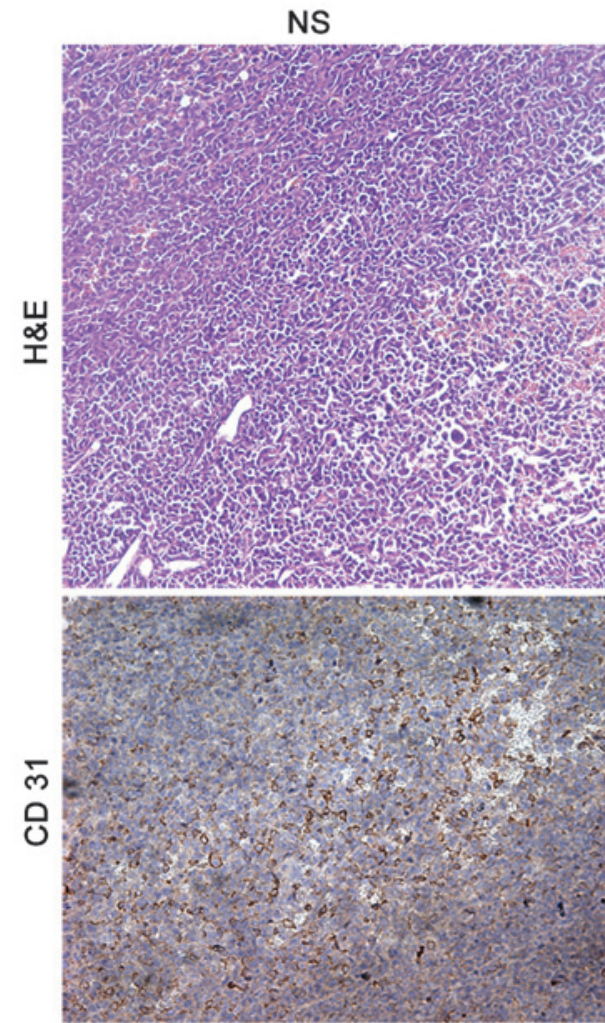

B

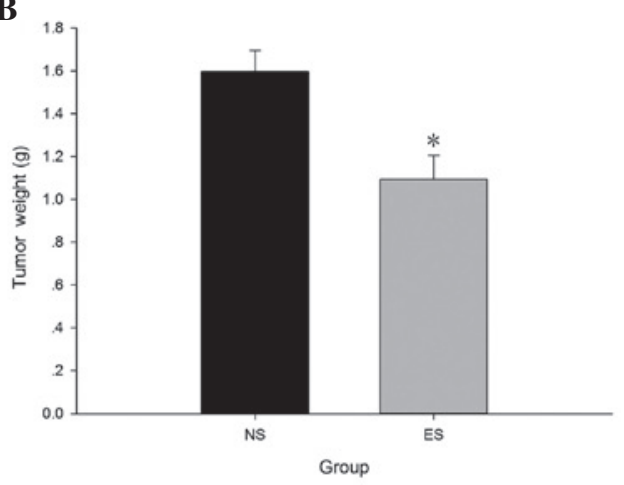

ES

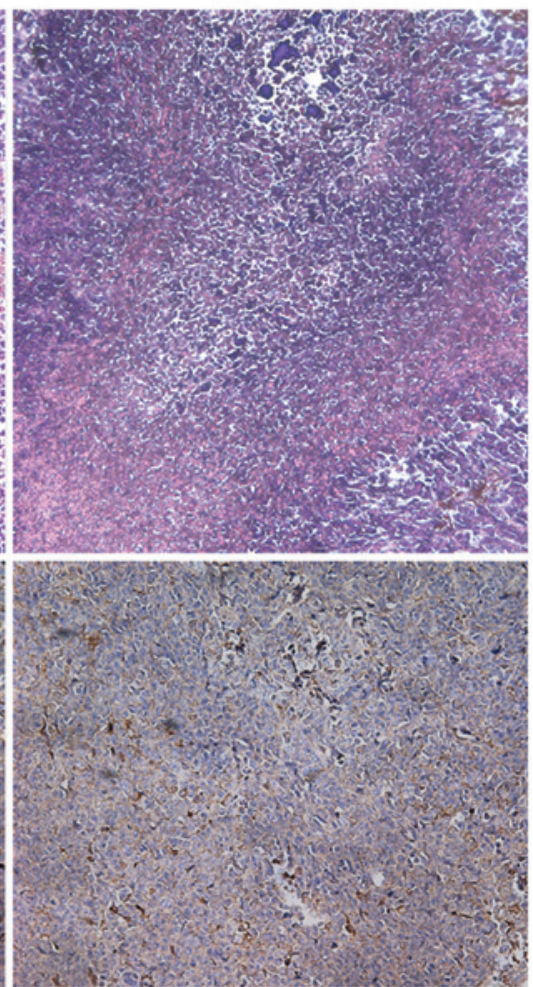

Figure 1. Effects of endostatin on tumor burden in Lewis lung carcinoma tumor-bearing mice. (A) Tumor lengths and widths on days 8-20. On day 14, the tumor weights of the ES group were significantly lower than the NS group. (B) Final tumor weights were calculated and compared using the independent sample test. (C) Tumors were stained with hematoxylin and eosin, and the vascular networks are shown using cluster of differentiation (CD)31 antibody staining (magnification, x200). Consistent with the increase in regional necrosis, vessel density significantly decreased following endostatin administration. The data are shown as the mean \pm standard deviation. " $\mathrm{P}<0.05$ vs. NS group. ES, endostatin group; NS, normal saline control group.

the NS group $(\mathrm{P}<0.05)$. Once the mice had been sacrificed on day 20, the tumors were removed and accurately weighed (Fig. 1B). The tumor weight of the ES group was significantly lower than the NS group $(\mathrm{P}<0.05)$. Immunohistochemical analysis of the tumor xenografts using the anti-CD31 antibody showed that the blood vessels appeared as primitive and dilated sinusoidal vascular structures that consisted of disorganized and tortuous vascular plexuses (Fig. 1C). The tumor xenografts of the ES group demonstrated clear blood vessels in comparison with the NS group, without expanded vascular plexuses, indicating that endostatin inhibits tumor angiogenesis. In addition, $H \& E$ revealed the prominent visibility of cell necrosis in the tumors of the ES group (Fig. 1C), which is a common feature that can be observed in tumors following endostatin administration $(14,15)$.
Effect of endostatin on body weight loss. Body weight was measured every 3 days once the tumors became palpable (Fig. 2A). The tumor-bearing mice gained weight early, but their weight significantly declined after day $14(\mathrm{P}<0.05)$. However, weight loss in the endostatin-treated mice was substantially lower than that in the NS mice $(\mathrm{P}<0.05)$. The NS mice weighed significantly less than the mice in the ES and normal groups $(\mathrm{P}<0.05)$. There was no significant difference in body weight between the endostatin-treated and normal mice. The tumors were removed, and the final body weights were measured after sacrifice (Fig. 2B).

Effect of endostatin on preventing anemia. Gross examination of the tumor-bearing mice revealed severe anemia, which manifested as noticeable paleness on the hairless regions 
A

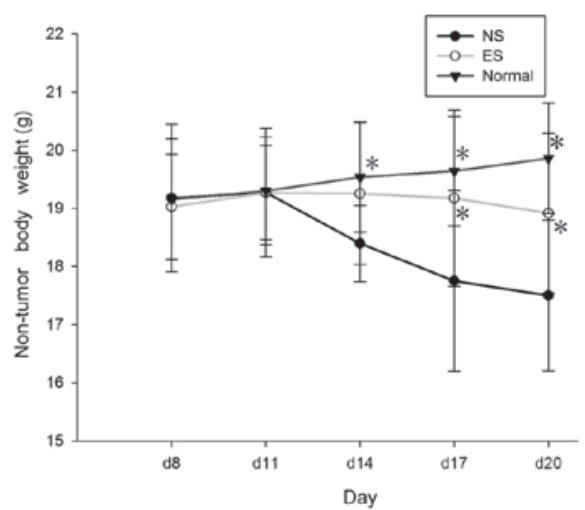

B

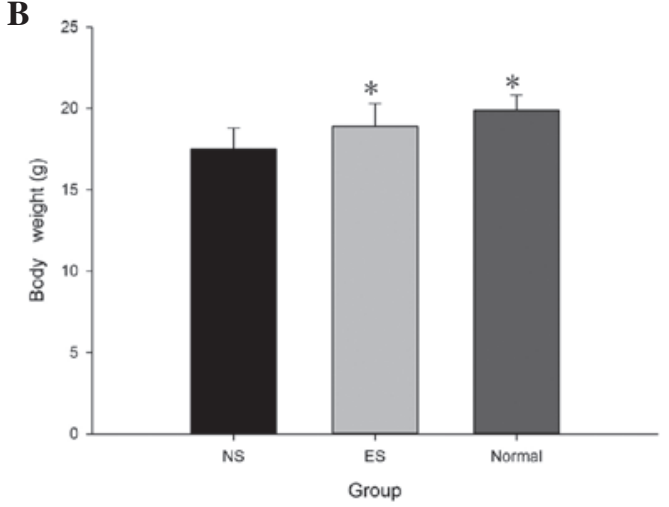

Figure 2. Effect of endostatin on body weight. (A) Body weight is expressed as total weight minus tumor weight. After 14 days, the body weights of the ES and normal groups were significantly higher than the NS group. (B) Final body weights were determined and compared. Data are shown as the mean \pm standard deviation. ${ }^{*} \mathrm{P}<0.05$ vs. NS group. ES, endostatin group; NS, normal saline control group.

A
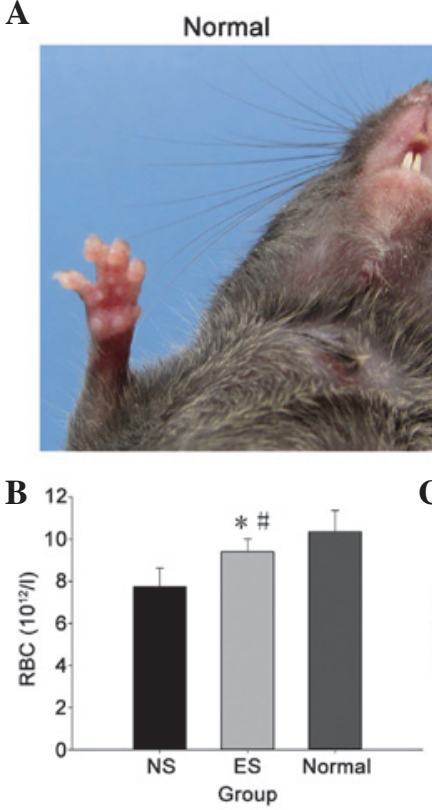

NS

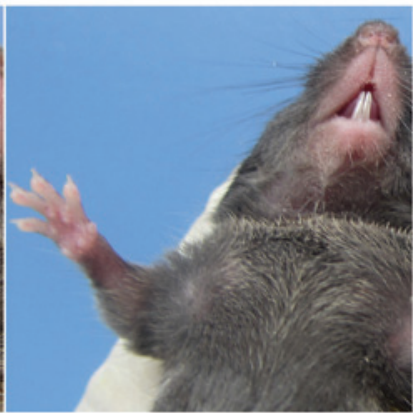

C 60

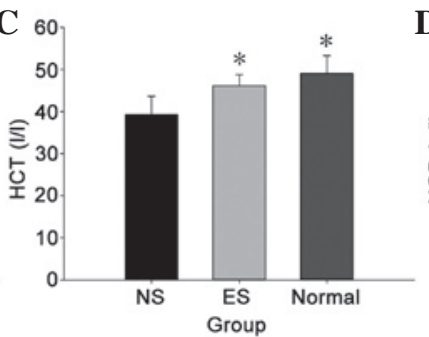

ES

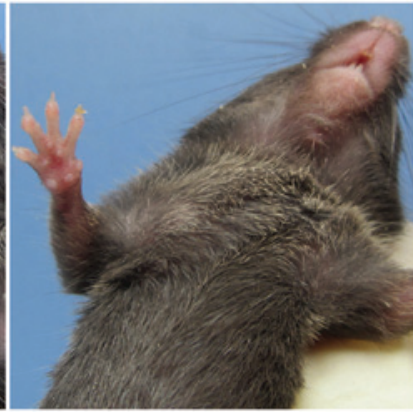

D 180

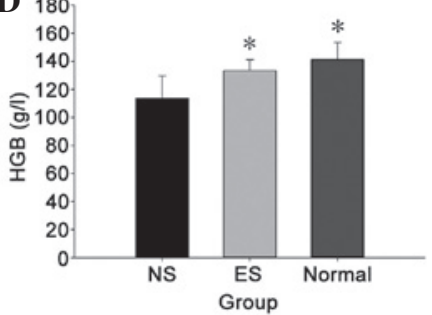

Figure 3. Effects of endostatin on preventing anemia. (A) After 12 days of treatment, images of a representative mouse from each group were captured to show that severe anemia had developed in the tumor-bearing mice. Blood samples were collected and (B) red blood cell (RBC) count, (C) hematocrit (HCT) and (D) hemoglobin (HGB) level were assessed. Data are shown as the mean \pm standard deviation. ${ }^{\#} \mathrm{P}<0.05$ vs. normal group. ${ }^{\mathrm{P}}<0.05$ vs. NS group. ES, endostatin group; NS, normal saline control group.

of the paws, mouth, nose and genitals (Fig. 3A). Hematological analysis of the peripheral blood revealed a significant decrease in HCT at day 20 after tumor implantation $(\mathrm{P}<0.05)$ (Fig. 3C). HGB and erythrocytes also significantly decreased in the peripheral blood $(\mathrm{P}<0.05)$ (Fig. 3B and D). These results showed that the tumor-bearing mice were severely anemic. However, endostatin prevented anemia to a certain degree in comparison with the NS group, and there were no significant differences in HCT or HGB between the ES group and the normal mice.

Endostatin induces changes in biochemical parameters. Certain biochemical parameters of liver function and metabolism were also analyzed. The LLC tumors significantly changed the majority of biochemical parameters in the NS and ES groups, in comparison with the normal group (Table I). For example, in comparison with the normal group, the levels of serum TP and ALB markedly decreased $(\mathrm{P}<0.05)$, and the levels of $\mathrm{CHO}$ and HDL markedly increased in the tumor-bearing mice $(\mathrm{P}<0.05)$. However, these changes in the tumor-bearing mice were somewhat alleviated by endostatin. There were also significant differences in terms of AST and $\mathrm{CHO}$ levels between the ES and NS groups.

Effects of endostatin on body weight and on pathological changes in certain organs. The dissected organs were weighed (Table II), and hepatosplenomegaly and abnormal kidney weight were noted in the tumor-bearing mice. The weights of the spleens in the tumor-bearing mice increased almost 2-fold in comparison with the normal group $(\mathrm{P}<0.05)$. However, endostatin improved the hepatosplenomegaly. The liver and kidney weights were significantly reduced $(\mathrm{P}<0.05)$ and reverted almost to those of the normal mice following endostatin administration. 
A
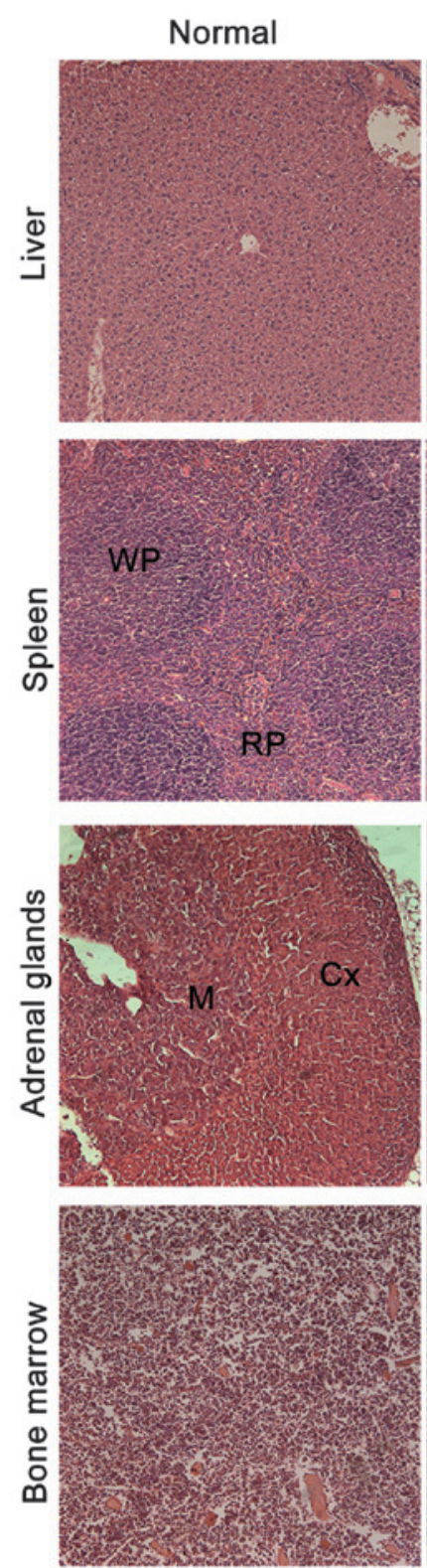

B

Normal
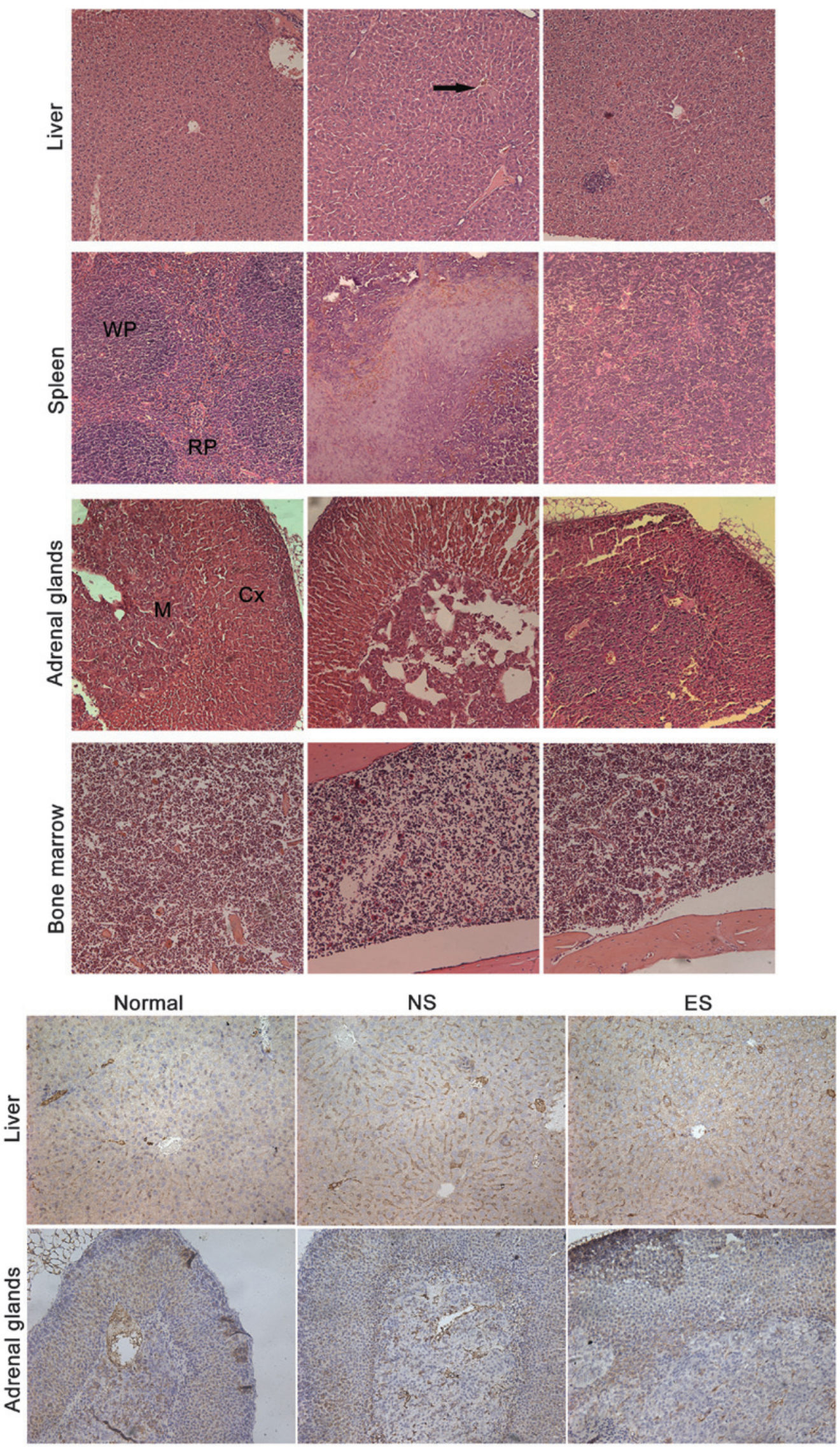

NS
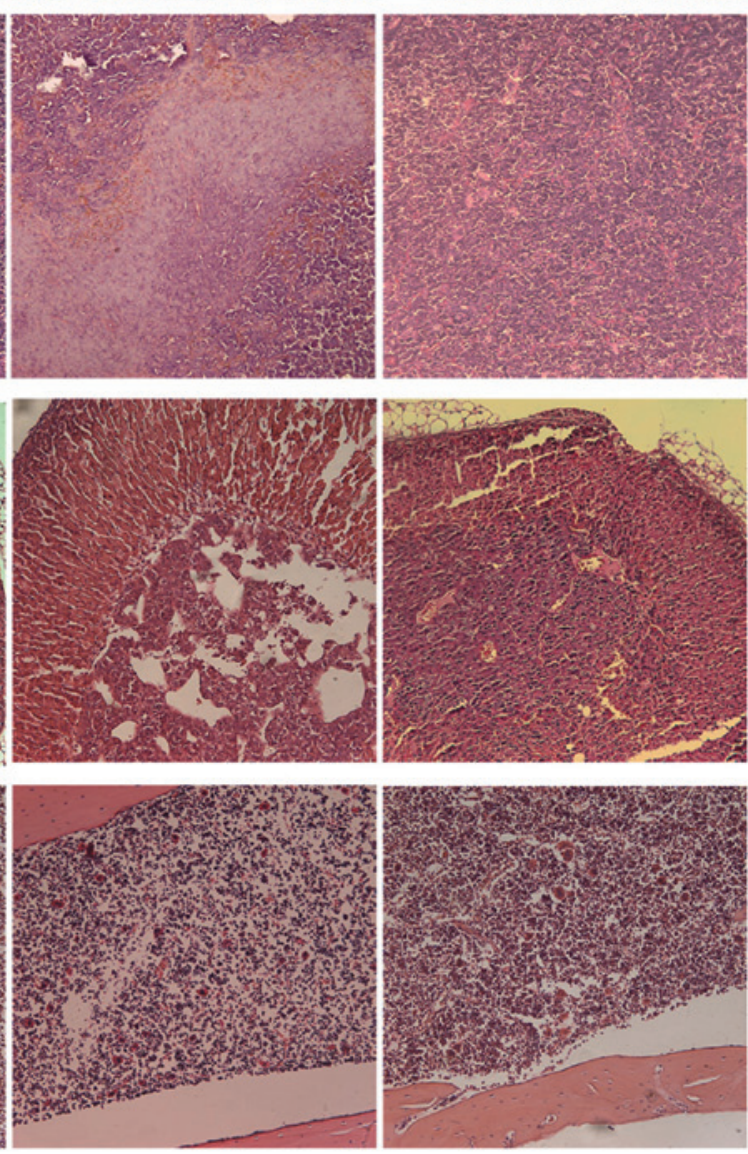

Figure 4. Histological analysis of the liver, spleen, adrenal glands and bone marrow (magnification, x200). (A) Tissue sections from the liver, spleen, bone marrow and adrenal glands stained with hematoxylin and eosin. The black arrow indicates the expanding vessels in the liver. (B) Tissue sections from the liver and adrenal glands stained with anti-cluster of differentiation 31 antibody. WP, white pulp; RP, red pulp; Cx, cortex; M, medulla; ES, endostatin group; NS, normal saline control group. 
Table I. Biochemical parameters.

\begin{tabular}{lccc}
\hline Parameters & Normal & NS & ES \\
\hline ALT, IU/1 & $51.20 \pm 16.35$ & $55.50 \pm 13.44$ & $46.70 \pm 7.65$ \\
AST, IU/1 & $133.20 \pm 15.70$ & $263.10 \pm 69.67^{\mathrm{a}}$ & $198.90 \pm 41.30^{\mathrm{a}, \mathrm{b}}$ \\
$\mathrm{TP}, \mathrm{g} / \mathrm{l}$ & $54.67 \pm 2.90$ & $50.59 \pm 2.31^{\mathrm{a}}$ & $50.28 \pm 3.26^{\mathrm{a}}$ \\
$\mathrm{ALB}, \mathrm{g} / \mathrm{l}$ & $28.57 \pm 1.95$ & $26.70 \pm 1.51^{\mathrm{a}}$ & $26.84 \pm 1.81^{\mathrm{a}}$ \\
$\mathrm{GLU}, \mathrm{mmol} / \mathrm{l}$ & $4.58 \pm 0.91$ & $5.92 \pm 1.88^{\mathrm{a}}$ & $6.37 \pm 1.06^{\mathrm{a}}$ \\
$\mathrm{CHO}, \mathrm{mmol} / \mathrm{l}$ & $1.23 \pm 0.27$ & $1.93 \pm 0.29^{\mathrm{a}}$ & $1.58 \pm 0.29^{\mathrm{a}, \mathrm{b}}$ \\
TG, $\mathrm{mmol} / \mathrm{l}$ & $0.60 \pm 0.12$ & $1.01 \pm 0.52^{\mathrm{a}}$ & $0.97 \pm 0.28^{\mathrm{a}}$ \\
HDL, mmol/l & $0.41 \pm 0.15$ & $0.63 \pm 0.10^{\mathrm{a}}$ & $0.60 \pm 0.10^{\mathrm{a}}$ \\
LDL, mmol/l & $0.16 \pm 0.04$ & $0.22 \pm 0.08^{\mathrm{a}}$ & $0.17 \pm 0.05$
\end{tabular}

Data are shown as the mean \pm standard deviation. ${ }^{a} \mathrm{P}<0.05$ vs. normal group. ${ }^{\mathrm{b}} \mathrm{P}<0.05$ vs. NS group. ALT, alanine aminotransferase; AST, aspartate aminotransferase; ALB, albumin; TP, total protein; GLU, glucose; TG, triglycerides; CHO, cholesterol; HDL, high-density lipoprotein; LDL, low-density lipoprotein; ES, endostatin group; NS, normal saline control group.

Table II. Organ weights.

\begin{tabular}{llrr}
\hline Organs & Normal,g & NS,g & ES,g \\
\hline Liver & $0.803 \pm 0.044$ & $1.055 \pm 0.144^{\mathrm{a}}$ & $0.85 \pm 0.079^{\mathrm{b}}$ \\
Spleen & $0.091 \pm 0.012$ & $0.159 \pm 0.037^{\mathrm{a}}$ & $0.143 \pm 0.037^{\mathrm{a}}$ \\
Kidney & $0.188 \pm 0.008$ & $0.209 \pm 0.025^{\mathrm{a}}$ & $0.198 \pm 0.016$ \\
\hline
\end{tabular}

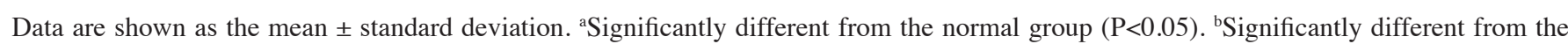
NS group $(\mathrm{P}<0.05)$. ES, endostatin group; NS, normal saline control group.

H\&E staining demonstrated that the hepatic sinusoid and hepatic central vein in the tumor-bearing mice exhibited a high degree of dilation architecture. In the spleens of the tumor-bearing mice, the white pulp (WP) and red pulp (RP) margins disappeared due to regional necrosis (Fig. 4A). The dilated sinusoidal blood vessels in the liver appeared normal in the ES group in comparison with the NS group. Similarly, the structure of the spleen was normalized by endostatin treatment. The adrenal tissue was dense without sinusoid dilation, and the bone marrow hematopoietic cells were rich and evenly distributed; there were no significant differences between the groups in terms of tissue structure of the adrenal glands or bone marrow.

Immunohistochemical analysis using the anti-CD31 antibody showed that the hepatic sinusoid and hepatic central vein of the tumor-bearing mice in the NS group exhibited primitive and dilated sinusoidal vascular structures (Fig. 4B). Treating these tumor-bearing mice with endostatin almost completely reversed the dilation of the sinusoidal blood vessels. The adrenal tissue structure was dense without sinusoid dilation, and there were no significant differences between the groups.

Endostatin inhibits IL- 6 and VEGF production. TNF- $\alpha$, IL-6 and VEGF expression in the sera and tumors were detected using ELISA. TNF- $\alpha$, IL- 6 and VEGF levels were significantly higher in the tumor-bearing mice compared with the normal group $(\mathrm{P}<0.05)$ (Fig. 5). IL-6 and VEGF production were significantly inhibited by endostatin, with no significant effect on TNF- $\alpha$ expression.

\section{Discussion}

The present study found that the body weights of LLC tumor-bearing mice quickly decline at 14 days post-tumor implantation. Gross examination revealed severe anemia, which manifested as considerable paleness in several hairless regions, including the paws, mouth, nose and genitals. Hematological analysis of the peripheral blood samples revealed a significant decrease in HCT at day 20 post-tumor implantation. HGB level and RBC count was also significantly decreased in the peripheral blood. These results showed that the tumor-bearing mice had developed severe anemia. Serum parameters that reflect hepatic function and carbohydrate, lipid and protein metabolism were already considerably disordered, however, the tumor-bearing mice also developed hepatosplenomegaly. Histological analysis demonstrated that high-density sinusoidal vasculature filled the entire liver. In the spleen, the WP and RP margins disappeared, accompanied by regional necrosis. In short, all these findings showed that the tumor-bearing mice suffered from CASS.

Endostatin, an endogenous broad-spectrum angiogenesis inhibitor, can selectively target neovascular endothelial cells and suppress tumor growth (16). The present study found 

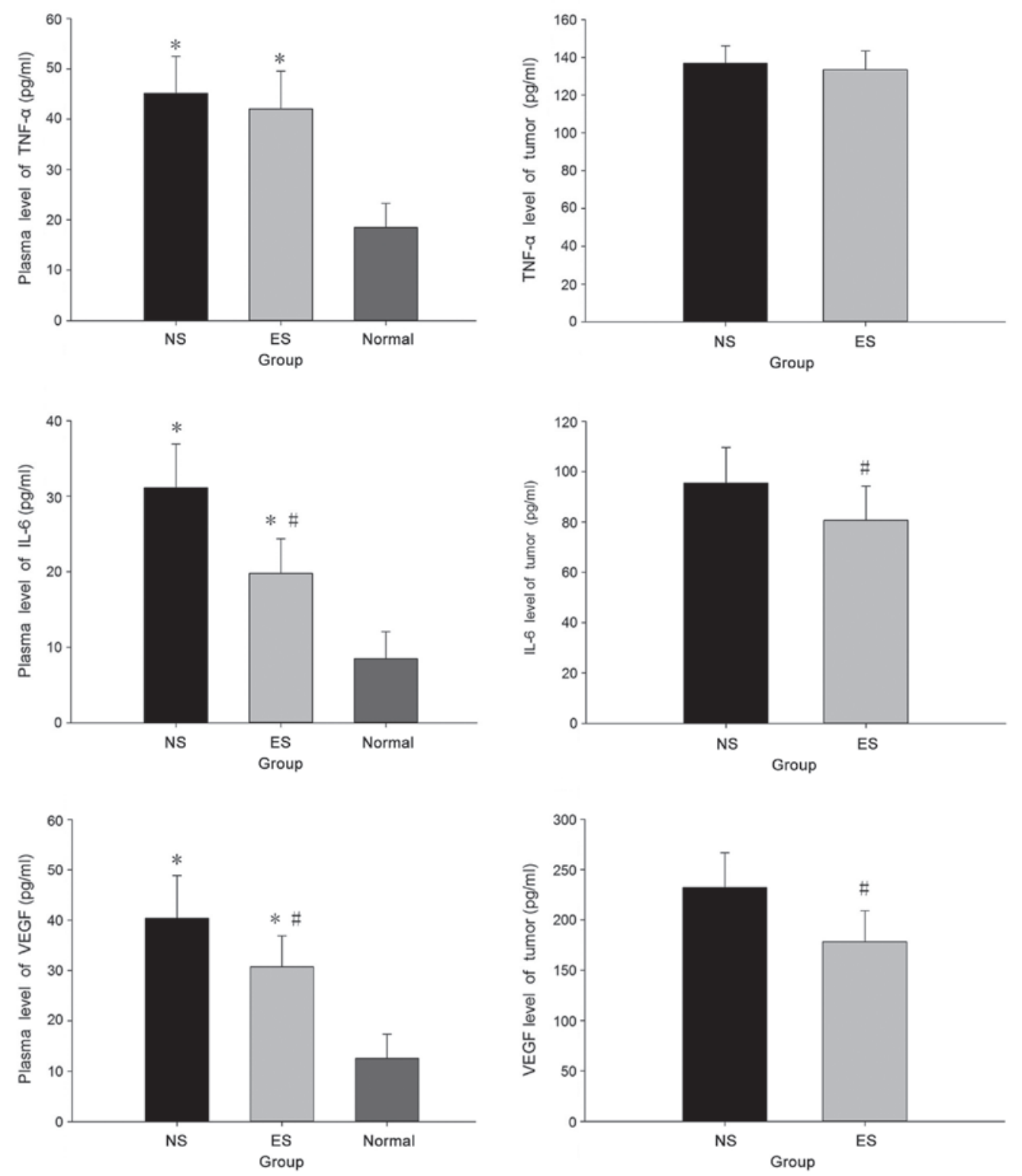

Figure 5. Effect of endostatin on serum and TNF- $\alpha$, IL-6 and VEGF cytokines. High IL-6 and VEGF levels were inhibited by endostatin, and no significant effect on TNF- $\alpha$ was observed. Data are shown as the mean \pm standard deviation. ${ }^{*} \mathrm{P}<0.05$ vs. control group. ${ }^{*} \mathrm{P}<0.05$ vs. NS group. ES, endostatin group; NS, normal saline control group; TNF- $\alpha$, tumor necrosis factor $\alpha$; IL-6, interleukin 6; VEGF, vascular endothelial growth factor.

that the tumor burden in the endostatin-treated mice was significant lower than in the placebo-treated mice. Furthermore, the CASS-alleviating effect of endostatin in the LLC tumor-bearing mice was evident by the weight gain, improvement in blood biochemical parameters and anemia, and the preservation of organ function. All these findings confirmed that endostatin can effectively affect off-tumor organs and improve CASS in an LLC tumor-induced murine model. Other antiangiogenic drugs, including bevacizumab, sunitinib and sorafenib, may also physiologically alter organ function and vascular remodeling, thereby improving CASS and progression-free survival in tumor-bearing mice (9). However, as the drugs are exogenous angiogenesis inhibitors, a large number of cancer patients develop adverse effects in response to antiangiogenic agents, including impaired wound healing, hypothyroidism, hypertension, hemorrhage, proteinuria and cardiovascular disorders $(9,17)$. Therefore, endostatin, which does not cause these adverse effects, could be used to treat patients with CASS.

Although certain cytokines, such as VEGF, TNF- $\alpha$ and IL-6, contribute to CASS, its underlying molecular mechanisms remain unknown $(18,19)$. For example, CASS is associated with an elevated serum IL-6 level, and anti-IL-6 monoclonal antibody therapy decreases the incidence of cancer-related anorexia and cachexia (1). TNF- $\alpha$ treatment can impair the oxidation of long-chain fatty acids (20). Cancer cachexia is partly mediated by spleen-secreted IL-6 (21), and can be ameliorated by L-carnitine via the regulation of serum TNF- $\alpha$ and IL- 6 levels, and the modulation of carnitine palmitoyltransferase expression and activity in the liver (22). Consistent with the results from these aforementioned studies, the present study found that serum VEGF, TNF- $\alpha$, and IL- 6 levels markedly increase with the progression of CASS, and that serum VEGF and IL-6 levels notably 
decrease following administration of endostatin. Therefore, it is possible that endostatin ameliorates CASS by attenuating serum VEGF and IL-6 levels, although the exact mechanism remains to be elucidated.

The majority of preclinical and clinical studies on anti-VEGF agents focus on tumor vasculature or growth, and little is known with regard to the systemic effects of these therapeutic agents. In preclinical studies, the systemic administration of antiangiogenic agents in non-tumor-bearing mice has been reported to alter vascular density and architecture in numerous organs and tissues, particularly endocrine organs containing fenestrated vessels (23). A previous study reported that the level of circulating VEGF correlates with the severity of CASS in tumor-bearing mice and human cancer patients, and that anti-VEGF agents could improve organ function (8). As a consequence of the off-tumor target effects, improvements to organ function and alterations in chemotoxic tolerance may be the most important mechanisms with a survival benefit in cancer patients with CASS $(8,24)$.

In summary, the present data showed that in an LLC tumor-induced CASS model, endostatin effectively reduced the tumor burden, preserved body weight and improved CASS, possibly by reducing the serum VEGF and IL-6 levels. Further studies are required to further explore the application of this endogenous angiogenesis inhibitor in the treatment of advanced cancer patients with CASS.

\section{Acknowledgements}

This study was supported by a grant from the National Natural Science Foundation of China (nos. 81071864 and 81372560). The authors would like to acknowledge the animal care provided by Mr. Yan-yang Liu and Mr. Li Wang.

\section{References}

1. Nathanson L and Hall TC: Introduction: paraneoplastic syndromes. Semin Oncol 24: 265-268, 1997.

2. Nakamura I, Shibata M, Gonda K, et al: Serum levels of vascular endothelial growth factor are increased and correlate with malnutrition, immunosuppression involving MDSCs and systemic inflammation in patients with cancer of the digestive system. Oncol Lett 5: 1682-1686, 2013.

3. Tisdale MJ: Cachexia in cancer patients. Nat Rev Cancer 2: $862-871,2002$

4. Inui A: Cancer anorexia-cachexia syndrome: current issues in research and management. CA Cancer J Clin 52: 72-91, 2002.

5. Takahashi Y, Yasumoto K and Mai M: Chemotherapy under cachectic conditions and the possibility of cachexia-controlled chemotherapy. Oncol Rep 14: 135-140, 2005.

6. Krzystek-Korpacka M, Matusiewicz M, Diakowska D, et al: Impact of weight loss on circulating IL-1, IL-6, IL-8, TNF-alpha, VEGF-A, VEGF-C and midkine in gastroesophageal cancer patients. Clin Biochem 40: 1353-1360, 2007.
7. Ebos JM, Lee CR, Christensen JG, et al: Multiple circulating proangiogenic factors induced by sunitinib malate are tumor-independent and correlate with antitumor efficacy. Proc Natl Acad Sci USA 104: 17069-17074, 2007.

8. Xue Y, Religa P, Cao R, et al: Anti-VEGF agents confer survival advantages to tumor-bearing mice by improving cancer-associated systemic syndrome. Proc Natl Acad Sci USA 105: 18513-18518, 2008.

9. Cao Y: Off-tumor target - beneficial site for antiangiogenic cancer therapy? Nat Rev Clin Oncol 7: 604-608, 2010.

10. Ling Y, Yang Y, Lu N, et al: Endostar, a novel recombinant human endostatin, exerts antiangiogenic effect via blocking VEGF-induced tyrosine phosphorylation of KDR/Flk-1 of endothelial cells. Biochem Biophys Res Commun 361: 79-84, 2007.

11. Zhang Y, Zhang J, Jiang D, et al: Inhibition of T-type $\mathrm{Ca}^{2+}$ channels by endostatin attenuates human glioblastoma cell proliferation and migration. Br J Pharmacol 166: 1247-1260, 2012.

12. Lim J, Duong T, Lee G, et al: The effect of intracellular protein delivery on the anti-tumor activity of recombinant human endostatin. Biomaterials 34: 6261-6271, 2013.

13. Xu W, Ye P, Li Z, et al: Endostar, a recently introduced recombinant human endostatin, inhibits proliferation and migration through regulating growth factors, adhesion factors and inflammatory mediators in choroid-retinal endothelial cells. Mol Biol (Mosk) 44: 664-670, 2010.

14. Huszthy PC, Brekken C, Pedersen TB, et al: Antitumor efficacy improved by local delivery of species-specific endostatin. J Neurosurg 104: 118-128, 2006.

15. Zhang X, Yang G, Zhang Y, et al: An experimental research into endostatin microbubble combined with focused ultrasound for anti-tumor angiogenesis in colon cancer. Gastroenterol Rep (Oxf) 2: 44-53, 2014.

16. Ning T, Jiang M, Peng Q, et al: Low-dose endostatin normalizes the structure and function of tumor vasculature and improves the delivery and anti-tumor efficacy of cytotoxic drugs in a lung cancer xenograft murine model. Thoracic Cancer 3: 229-238, 2012.

17. Jain RK: Normalization of tumor vasculature: an emerging concept in antiangiogenic therapy. Science 307: 58-62, 2005.

18. Kayacan O, Karnak D, Beder S, et al: Impact of TNF-alpha and IL-6 levels on development of cachexia in newly diagnosed NSCLC patients. Am J Clin Oncol 29: 328-335, 2006.

19. Yang Q, Wan L,Zhou Z, et al: Parthenolide from Parthenium integrifolium reduces tumor burden and alleviate cachexia symptoms in the murine CT-26 model of colorectal carcinoma. Phytomedicine 20: 992-998, 2013.

20. Noguchi Y, Makino T, Yoshikawa T, et al: The possible role of TNF-alpha and IL-2 in inducing tumor-associated metabolic alterations. Surg Today 26: 36-41, 1996.

21. Barton BE and Murphy TF: Cancer cachexia is mediated in part by the induction of IL-6-like cytokines from the spleen. Cytokine 16: 251-257, 2001.

22. Liu S, Wu HJ, Zhang ZQ, et al: L-carnitine ameliorates cancer cachexia in mice by regulating the expression and activity of carnitine palmityl transferase. Cancer Biol Ther 12: 125-130, 2011.

23. Kamba T, Tam BY, Hashizume H, et al: VEGF-dependent plasticity of fenestrated capillaries in the normal adult microvasculature. Am J Physiol Heart Circ Physiol 290: H560-H576, 2006.

24. Rein DT, Volkmer AK, Volkmer J, et al: Systemic administration of bevacizumab prolongs survival in an in vivo model of platinum pre-treated ovarian cancer. Oncol Lett 3: 530-534, 2012. 